\title{
RETRIEVAL OF KINETIC RATES IN REACTIONS WITH SEMI BATCH LIQUID PHASE USING ILL-POSED INVERSE PROBLEM THEORY
}

\section{Rita C. O. Sebastião* e João Pedro Braga}

Departamento de Química, Instituto de Ciências Exatas, Universidade Federal de Minas Gerais, Av. Antônio Carlos, 6627, 31270-901

Belo Horizonte - MG, Brasil

\section{Luciano S. Virtuoso}

Curso de Química, Centro de Ciências Exatas, Universidade Federal de Alfenas, Rua Gabriel Monteiro da Silva, 700, $37130-000$ Alfenas - MG, Brasil

Emílio Borges

Departamento de Química, Centro de Ciências Exatas e Tecnológicas, Universidade Federal de Viçosa, Av. P. H. Rolfs, 36570-000 Viçosa - MG, Brasil

Recebido em 21/1/10; aceito em 20/9/10; publicado na web em 8/12/10

\begin{abstract}
A neural network procedure to solve inverse chemical kinetic problems is discussed in this work. Rate constants are calculated from the product concentration of an irreversible consecutive reaction: the hydrogenation of Citral molecule, a process with industrial interest. Simulated and experimental data are considered. Errors in the simulated data, up to $7 \%$ in the concentrations, were assumed to investigate the robustness of the inverse procedure. Also, the proposed method is compared with two common methods in nonlinear analysis; the Simplex and Levenberg-Marquardt approaches. In all situations investigated, the neural network approach was numerically stable and robust with respect to deviations in the initial conditions or experimental noises.
\end{abstract}

Keywords: kinetic; ill-posed inverse problem; dynamical optimization.

\section{INTRODUCTION}

The process of obtaining physical information from experimental data is an ill-posed inverse problem if one of the three conditions, existence, uniqueness and continuity with respect to experimental noises, is not satisfied. ${ }^{1}$ Usually, robust techniques are required to solve this kind of problem which appears in several scientific and technological areas. For example, calculation of kinetic parameters from experimental properties related to chemical concentrations is called inverse kinetic problem. Methods of parameters estimation such as rank annihilation factor analysis, ${ }^{2}$ curve resolution methods, ${ }^{3}$ Monte $\mathrm{Carlo}^{4}$ or control type technique ${ }^{5}$ have been extensively described in literature to estimate kinetic constants. A common difficulty in all these techniques is the sensitivity with respect to experimental random noises of the derivative operators, used to model the kinetic processes. This fact is sufficient to classify the problem as an ill-posed kinetic inverse problem. ${ }^{6}$

The work to be developed here consists in an application of a stable numerical method to deal ill-posed kinetic problem. Recently a general approach based on recurrent neural networks was proposed to solve this kind of problem. ${ }^{7,8}$ This technique has also been used successfully to study magnetic resonance multiple sclerosis diagnostic, ${ }^{9}$ retrieval of probability density function from experimental positron annihilation lifetime spectra, ${ }^{10}$ for the inversion of intermolecular potential ${ }^{11}$ and to calculate molecular force fields from experimental vibrational frequencies. ${ }^{12}$

The neural network technique can also be applied to a kinetic problem of industrial interest which involves irreversible consecutive reactions: the hydrogenation of the Citral molecule. This molecule

*e-mail: ritacos@ufmg.br contains different reaction sites and the hydrogenation process is described by a complex kinetic model, although parallel reactions, like acetalization and cyclization can also occur. Increasing the catalyst density in the reaction medium, by drainage of the liquid phase, will enhance the principal reactions speed. As a consequence, the stages of competitive reactions are delayed. ${ }^{13,14}$ Rate constants for the process under consideration are obtained from experimental concentrations of the final product.

A model system of four kinetic equations will be used to exemplify and emphasize the usefulness of the methods dealing with random noise in the concentrations. Experimental data will carry an inherent error and the preliminary analysis with synthetic data will be important to learn about inverting real laboratory data. Inverting data with noise is not possible to methods that use matrix procedure. The robustness of the neural network with respect to experimental noises is compared with the Simplex and the Levenberg-Marquardt methods, commonly used in nonlinear regression approaches..$^{15,16}$.

\section{THEORETICAL BACKGROUND}

\section{Citral hidrogenation kinetic modeling}

According to the Langmuir-Hinshelwood kinetics, catalytic hydrogenation of citral on nickel catalyst is an isothermal consecutive first order reaction and can be described as a competitive absorption model.${ }^{14}$ In this process, the citral (A component) reacts in three irreversible first-order reactions, in which the primary product citronellal, (B component), is formed very fast, whereas citronellol (C component) and 3,7-dimetyloctanol (D component) are formed in a comparative slowly way. This reaction sequence can be seen in Figure 1. ${ }^{13,14}$ 
<smiles>CC(C)=CCCC(C)C</smiles>
Citral cis<smiles>CC(C)=CCCC(C)=CC=O</smiles>
Citral trans

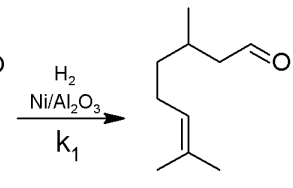

Citronellal<smiles>CC(C)=CCCC(C)CCO</smiles>

Citronellol

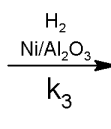

3,7-dimethyloctanol
Figure 1. Consecutive reactions for the Citral hidrogenation in $\mathrm{Ni}_{2} \mathrm{Al}_{2} \mathrm{O}_{3}$

To accelerate the secondary and tertiary products formation, a semi batch reactor concept has to be adopted. In this procedure, the catalyst-liquid ratio is increased with time by a constant volumetric flow out from the reactor, allowing the accurate kinetic parameters determination in a single experiment. ${ }^{13,14}$ In this semi batch reactor mode, as established in the references 13 and 14, the mass balance equation is

$$
\frac{d n_{i}}{d t}=r_{i} m_{c a t}-\dot{n}_{i, o u t}
$$

in which $n_{i}, r_{i}, m_{\text {cat }}$ and $\dot{n}_{i, o u t}$ are respectively the molar amount, the generation rate, the catalyst mass and the molar flow of $\mathrm{i}^{\text {th }}$ component. Due to an extensive stirring on the reactor, the concentrations in the continuously pumped volume flow and in the semi-batch reactor are constant and one can adopt $\dot{n}_{i, \text { out }}=n_{i}$ and also, $n_{i}=c_{i} V_{L}$, with $V_{L}=V_{0}-V_{T}$. Therefore, the Equation 1 can be rewritten as,

$$
\begin{gathered}
\frac{d\left(c_{i} V_{L}\right)}{d t}=r_{i} m_{\text {cat }}-c_{i} \dot{V}_{\text {out }} \\
\frac{d c_{i}}{d t}=r_{i} \frac{m_{\text {cat }}}{V_{L}}-\frac{d V_{L}}{d t} \frac{c_{i}}{V_{L}}-\frac{c_{i} \dot{V}_{\text {out }}}{V_{L}}
\end{gathered}
$$

and in a simplified form,

$$
\frac{d c_{i}}{d t}=r_{i} \frac{m_{c a t}}{V_{L}}=r_{i} \rho
$$

since $d V_{L} / d_{t}=-V_{\text {out }}$ and the variable bulk density of the catalyst $\left(V_{0}\right.$ is the initial volume of the liquid fase, $V$ the volumetric flow and $t$ the time of the reaction).

The generation rates, $r_{i}$, to the citral hydrogenation can be obtained considering the competitive adsorption model in which all the reactants are adsorbed on the same active sites and molecular hydrogen is also adsorbed. This scheme can be written as:

$$
\begin{aligned}
& P_{i}+{ }^{*} \stackrel{K_{i}, a c t}{\longrightarrow} P_{i}^{*} \\
& H_{2}+{ }^{*} \stackrel{K_{H}}{\longrightarrow} H_{2}^{*} \\
& P_{i}^{*}+H_{2}^{*} \stackrel{k_{i}}{\longrightarrow} P_{i+1}^{*}+* \\
& P_{i+1}^{*} \stackrel{K_{i+1}}{\longrightarrow} P_{i+1}+*
\end{aligned}
$$

in which $*$ is the active site, $P_{i}$ and $P_{i+1}$ are consecutive hydrogenation products. In this representation, hydrogenation reactions can be assumed as irreversible and rate-determining steps, deriving the rate Equations 4:

$$
\begin{aligned}
& r_{1}=\frac{k_{1} K_{A} C_{A}}{1+K_{A} C_{A}+K_{B} C_{B}+K_{C} C_{C}+K_{D} C_{D}} \frac{K_{H} C_{H}}{1+K_{H} C_{H}} \\
& r_{2}=\frac{k_{2} K_{B} C_{B}}{1+K_{A} C_{A}+K_{B} C_{B}+K_{C} C_{C}+K_{D} C_{D}} \frac{K_{H} C_{H}}{1+K_{H} C_{H}} \\
& r_{3}=\frac{k K C_{C} \quad C}{1+K_{A} C_{A}+K_{B} C_{B}+K_{C} C_{C}+K_{D} C_{D}} \frac{K_{H} C_{H}}{1+K_{H} C_{H}}
\end{aligned}
$$

with $C_{H}, K_{i}$ and $k_{i}$ the hydrogen concentration, the adsorption constants and the reaction rate constants, respectively. However, it was shown that citral hydrogenation is better represented considering the denominator equal to the unit in Equations $4 . .^{13,14}$ In this case, the adsorption of all components were neglected and the generation rates for the components,

$$
\begin{aligned}
& r_{A}=-r_{1} \\
& r_{B}=r_{1}-r_{2} \\
& r_{C}=r_{2}-r_{3} \\
& r_{D}=r_{3}
\end{aligned}
$$

can be substituted in Equations 2, resulting in the kinetic Equations 5:

$$
\begin{aligned}
& \frac{d C_{A}}{d t}=-k_{1}^{\prime} C_{A} C_{H} \rho \\
& \frac{d C_{B}}{d t}=\left(k_{1}^{\prime} C_{A}-k_{2}^{\prime} C_{B}\right) C_{H} \rho \\
& \frac{d C_{C}}{d t}=\left(k_{2}^{\prime} C_{B}-k_{3}^{\prime} C_{C}\right) C_{H} \rho \\
& \frac{d C_{D}}{d t}=k_{3}^{\prime} C_{C} C_{H} \rho
\end{aligned}
$$

Since $C_{H}$ is kept constant, the above coupled differential equation can be seen as a linear system on the concentrations, even for a time-varying parameter, $\rho$. Rate constants will be obtained from this kinetic model.

\section{Neural network theoretical background}

To obtain the citral hydrogenation kinetic rate constants from the product concentration data, an ill-posed inverse problem has to be solved. This is clear, if experimental data are treated as a first place. Taking derivative of concentrations will enlarge the measured inherent experimental error, for the experimental errors are random. This can give positive and negative derivate of the concentration. The objective of inverse kinetic problem is to handle this ill-posed problem. In this work, a methodology using recurrent neural network is applied. ${ }^{8}$ This approach is different from the one that uses a pre-defined function, since in this case errors are neglected.

Artificial neural networks are computer codes that simulate the human brain mechanism. While dealing with inverse problem, the purpose of neural networks is to find a solution of minimum norm and also one which produces a residual minimum norm. ${ }^{17-21}$ The Hopfield neural network (HNN) works as a dynamical system, which can be confirmed by its time derivative of the error function. Concepts like initial conditions, trajectory and constant of motion are also used in the algorithm, in the same way as in dynamical systems. Following Vemuri and Jang, an energy function is defined as ${ }^{17-21}$

$$
E=\frac{1}{2} \sum_{j=1}^{m}\left(C_{c a l, j}-C_{\mathrm{exp}, j}\right)^{2}
$$


with $C_{c a l}$ and $C_{\text {exp }}$ the calculated and experimental properties, respectively.

Since the calculated property, $C_{c a l}$, depends on the activated neurons, $f\left(u_{i}(\tau)\right.$, and the neurons states are changed during the learning time, $\tau$, the derivative of the energy function can be represented as ${ }^{19,21}$

$$
\frac{d E}{d \tau}=\sum_{i=1}^{n} \sum_{j=1}^{m}\left(e_{j} \frac{\partial\left(C_{c a l}\right)_{j}}{\partial f_{i}} \frac{\partial f_{i}}{\partial u_{i}} \frac{d u_{i}}{d \tau}\right)
$$

in which $e_{j}=\left(C_{\text {cal. } j}-C_{\text {exp.j }}\right)$ and $n$ is the number of neurons used in the network. This is also the number of variables to be retrieved from the inversion process from the $m$ experimental data. ${ }^{8}$

In the neural network algorithm, two conditions have to be imposed such that the error function is a decreasing function with respect to the learning time. The first one to be imposed is:

$$
\frac{d u_{i}}{d \tau}=-\sum_{j=1}^{m} \frac{\partial\left(C_{c a l}\right)_{j}}{\partial f_{i}} e_{j}
$$

or

$$
\frac{d u_{i}}{d \tau}=-\frac{\partial E}{\partial f_{i}}
$$

transforming (7) into

$$
\frac{d E}{d \tau}=-\sum_{i=1}^{n} \frac{\partial f_{i}}{\partial u_{i}}\left(\frac{d u_{i}}{d \tau}\right)^{2}
$$

An increasing activation function, that is, $\partial f_{i} / \partial u_{i}>0$, will make, from (9), the error function to be a decreasing function. This constitutes the second condition in the algorithm. This important property of the Hopfield network will guarantee an ever decreasing error during the learning process which is not satisfied by other algorithm, such as Simplex and Levenberg-Marquardat approaches.

In this algorithm, among infinite solutions, the network provides that one which best reproduces the experimental property. This property is not necessarily the variables of the system, but can be a function of them. For example, in the present work, the measured property is the chemical concentration, $C_{\text {exp }}$, and the variables (activated neurons) are the rate constants of the process, which vary with the network learning time, i.e during the minimization of energy, $E$. The evaluated property, $C_{c a l}$, is obtained by numerical integration of Equations 8 .

From a given initial guess, necessary to start the learning process, the set of differential equations, Equations 8, was integrated by a fourth order Runge-Kutta method, ${ }^{22}$ whereas the gradient were calculated by a two point central difference equation. Learning process is stopped at the point in which, $d u_{i} / d \tau=0$, the minimum of the error function.

Due to the dynamical character of the Hopfield neural network, inversion of matrix is not actually necessary. The neurons are adapted themselves to meet the objective function. This is another important neural network approach advantage. In extreme cases this recursive theory is capable to find the solution of even rank deficient cases. Associate with this point, the neural network does not require an analytical function to be fitted, as exemplified in the present work. This has also been discussed in another work in which functional solution was found. ${ }^{7}$

\section{RESULTS AND DISCUSSION}

\section{Analysis with simulated data}

The first part of this work was performed using simulated data to check the efficiency of the proposed method. For this procedure, analytical or numerical solutions of Equations 5 are necessary. To analytically solve these equations one can use the integrant factor theory, obtaining,

$$
\begin{gathered}
\frac{\mathrm{C}_{\mathrm{A}}}{\mathrm{C}_{\mathrm{A} 0}}=\left(1-\frac{t}{\tau_{0}}\right)^{-k_{1} C_{H} \rho_{0} \tau_{0}} \\
C_{B}=\frac{\mathrm{k}_{1}}{\left(\mathrm{k}_{2}-\mathrm{k}_{1}\right)}\left[\left(1-\frac{t}{\tau_{0}}\right)^{-k_{1} C_{H} \rho_{0} \tau_{0}}-\left(1-\frac{t}{\tau_{0}}\right)^{-k_{2} C_{H} \rho_{0} \tau_{0}}\right] \\
C_{C}=\frac{\mathrm{k}_{2} \mathrm{k}_{1}}{\left(\mathrm{k}_{2}-\mathrm{k}_{1}\right)\left(\mathrm{k}_{3}-\mathrm{k}_{1}\right)}\left(1-\frac{t}{\tau_{0}}\right)^{-k_{1} C_{H} \rho_{0} \tau_{0}} \\
-\frac{\mathrm{k}_{2} \mathrm{k}_{1}}{\left(\mathrm{k}_{2}-\mathrm{k}_{1}\right)\left(\mathrm{k}_{3}-\mathrm{k}_{2}\right)}\left(1-\frac{t}{\tau_{0}}\right)^{-k_{2} C_{H} \rho_{0} \tau_{0}} \\
+\frac{\mathrm{k}_{2} \mathrm{k}_{1}}{\left(\mathrm{k}_{3}-\mathrm{k}_{1}\right)\left(\mathrm{k}_{3}-\mathrm{k}_{2}\right)}\left(1-\frac{t}{\tau_{0}}\right)^{-k_{3} C_{H} \rho_{0} \tau_{0}}
\end{gathered}
$$

with $\rho=\frac{\rho_{0}}{1-\frac{\dot{V}}{V_{0}} t}=\frac{\rho_{0}}{1-\frac{t}{\tau_{0}}} ; \rho_{0}=m_{c a t} / V_{0}$ and $\tau_{0}=V_{0} / V$.

In Figure 2 Equations 10, 11 and 12 were confronted with experimental data at $50{ }^{\circ} \mathrm{C}$ with drainage of the liquid phase at hydrogen pressure of 10 bar. The experimental data and parameters were obtained from refs. 13, 14 and are presented in Table 1. The good agreement of these data confirms the reliability of the model.

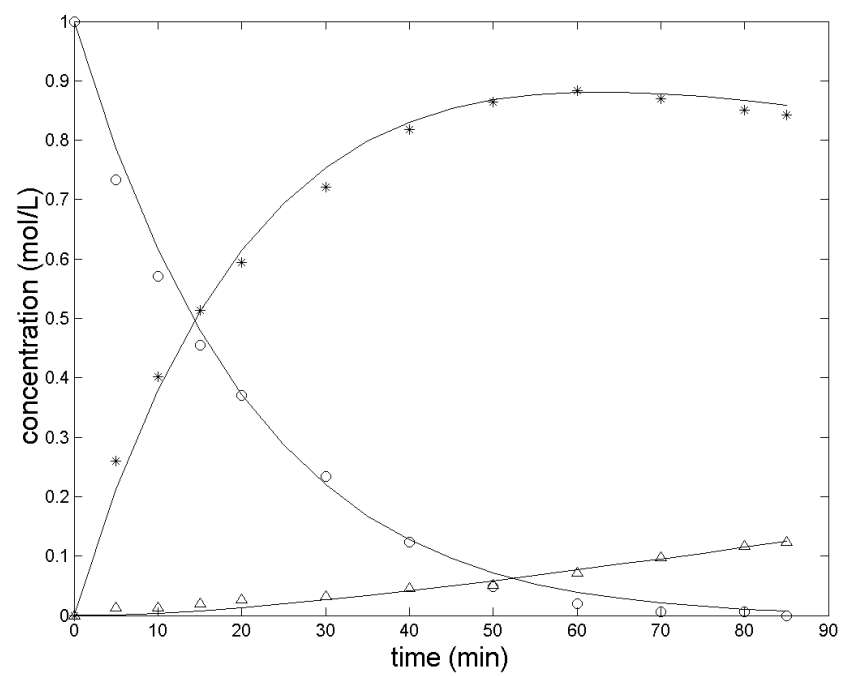

Figure 2. Experimental and simulated data at $50{ }^{\circ} \mathrm{C}$ with drainage of the liquid phase at hydrogen pressure of 10 bar. $^{13,14}$ Kinetic model is presented as full line and experimental data by symbols: ( $\mathrm{O}$ Citral, (*) Citronellal and $(\triangle)$ Citronellol

At this point it is important to emphasize that using analytical functions to fit the data implicitly assumes these are the correct functions, requiring a very large number of data, which is not usually the case. Therefore, one has, in general, an approximated solution to the problem. Rate constants obtained by the above procedure have a meaning only within this restriction. Methods such as LevenbergMarquardt and others least square approaches require, at some stage, inversion of a matrix. Applying this inverted matrix to the experimental data will cause large fluctuation on the results, due to the matrix condition, as discussed in ref. 18 for a model system. 
Table 1. Experimental parameters for citral hydrogenation at $50{ }^{\circ} \mathrm{C}$ and 10 bar. According to refs. 13 and 14

\begin{tabular}{cc}
\hline parameter & value \\
\hline$C_{0 A}$ & $0.102 \mathrm{~mol} / \mathrm{L}$ \\
$\mathrm{H}_{2}$ solubility & 0.00023 \\
$\rho_{0}$ & $1.54 \mathrm{~g} / \mathrm{L}$ \\
$\tau_{0}$ & $263.9 \mathrm{~min}$ \\
$k_{1}^{\prime}$ & $134 \mathrm{~L}^{2} / \mathrm{mol} \mathrm{min} \mathrm{m}$ \\
$k_{2}^{\prime}$ & $4.96 \mathrm{~L}^{2} / \mathrm{mol} \mathrm{ming}$ \\
$k^{\prime}$ & $4.42 \mathrm{~L}^{2} / \mathrm{mol} \mathrm{min} \mathrm{g}$
\end{tabular}

Also, retrieving kinetic models from experimental concentration data is a challenging subject in this area. The consecutive kinetic model analyzed here is a prototype, which allows a clear comparison among the most used techniques to deal with this kind of problem. Nevertheless, one may ask if this model is a correct one, for the given data. This kind of problem, which in the inverse problem area is known as an inverse problem to find the kernel, is a more difficult one. Further work along this line, adapting the present algorithm to find the kernel, can be carried out in the future.

Initial guesses for the rate constants are used as neural network inputs whereas the activated neuron states correspond to inverted rate constants. This initial condition is important to obtain converged results and can be used as a criterion to check the reliability of the computational method, since for an exact initial guess and theoretical data, activated neuron states can not change in the learning time. Different initial conditions, necessary to propagate the coupled differential equations, were tested in the procedure using simulated data. For example, if an initial guess of $10 \%$ away from the correct value is used, the inverted rate constants produce an error, $E=\frac{1}{2} \sum_{j=1}^{m}\left(C_{c a l, j}-C_{\exp , j}\right)^{2}$ of $1.68 \times 10^{-7}$ in relation to experimental Citronellol concentrations.

To test the algorithm stability, random noises in the range 1-10\% were added to the simulated concentrations. Even at a noise level of $10 \%$, the algorithm was stable, as shown in Table 2. In this case, the maximum error is $10.4 \%$ to the $\mathrm{k}_{2}$ constant and the error in the recovered concentration is $1.31 \times 10^{-7}$. In all these tests the error in recovered $\mathrm{k}_{1}$ was null.

Table 2. Analysis of random noise in the simulated concentration by neural network. Numbers in parentheses are for power of 10

\begin{tabular}{cccc}
\hline $\begin{array}{c}\text { Random noise added } \\
\text { in the concentration } \\
\%\end{array}$ & $\begin{array}{c}\text { \% error in } \\
\text { recovered } \mathrm{k}_{2}\end{array}$ & $\begin{array}{c}\text { \% error in } \\
\text { recovered } \mathrm{k}_{3}\end{array}$ & $\begin{array}{c}\text { Error in recovered } \\
\text { concentration }\end{array}$ \\
\hline 1 & 1.21 & 0 & $1.58(-9)$ \\
4 & 3.23 & 0.23 & $1.37(-8)$ \\
5 & 5.24 & 0.45 & $3.64(-8)$ \\
7 & 7.26 & 0.45 & $6.84(-8)$ \\
9 & 9.27 & 0.68 & $1.08(-7)$ \\
10 & 10.4 & 0.68 & $1.31(-7)$ \\
\hline
\end{tabular}

\section{Analysis with experimental data}

Citronellol experimental concentrations were also used in the inversion procedure. Necessary data to define this system are given in Table 1. If the constants listed in this table are taken as initial condition, from which the neural network is suppose to learn, the rate constants (units as in Table 1) recovered by the network are $\mathrm{k}_{1}=134$; $\mathrm{k}_{2}=4.97$ and $\mathrm{k}_{3}=4.43$, with a residual error of $6.75 \times 10^{-4}$.

The concentrations obtained in the direct problem by using these inverted rate constants are in better agreement with experimental concentrations than the simulated results obtained by the kinetic model, Equations 5. For initial guesses up to $10 \%$ far off those listed in Table 1 , the algorithm was also robust and the rate constants calculated are the same as presented in the previous paragraph. The neural network performance was compared to other optimization methods, usually employed in nonlinear regression such as Simplex and the LevenbergMarquardt algorithms..$^{15,16} \mathrm{~A}$ comparison between these methods and the neural network is shown in Tables 2-3 in which the robustness of the three methods was checked by adding random noises to the simulated concentrations.

Table 3. Analysis of random noise in the simulated concentration by Simplex and Levenberg-Marquardt algorithms

\begin{tabular}{cccccccccc}
\hline \multicolumn{3}{c}{$\begin{array}{c}\text { Levenberg-Marquardt } \\
\text { Random noise added in the } \\
\text { concentration }\end{array}$} & $\begin{array}{c}c \\
\text { Random noise added in the } \\
\text { concentration }\end{array}$ \\
$0 \%$ & $3 \%$ & $7 \%$ & $10 \%$ & $0 \%$ & $3 \%$ & $7 \%$ & $10 \%$ \\
\hline Error in $k_{1}^{\prime}(\%)$ & 0.1493 & 0.5970 & 0 & 0 & 0 & 0 & 0 & 0 \\
Error in $k_{2}^{\prime}(\%)$ & 0.1008 & 3.306 & 7.016 & 10.18 & 0 & 3.004 & 7.016 & 10.00 \\
Error in $k_{3}^{\prime}(\%)$ & 0.8597 & 1.425 & 7.737 & 7.805 & 0 & 3.325 & 7.805 & 11.19 \\
Error* & 4.220 & 2.555 & 1.809 & 7.055 & 3.914 & 2.117 & 2.509 & 2.538 \\
& $(-11)$ & $(-09)$ & $(-11)$ & $(-09)$ & $(-18)$ & $(-16)$ & $(-16)$ & $(-17)$ \\
\hline
\end{tabular}

*As Equation 6. Numbers in parentheses are for power of 10.

The neural network is more robust than the other algorithms investigated for all noise levels analyzed here. For example, the following average deviations in the rate constants with respect to its reference values are obtained considering different noises in the concentrations. For a noise error of $3 \%$, the neural network approach returns an error of $1.6 \%$ in the inverted rate constants, whereas the recovery for the Simplex and Levenberg-Marquardt are 122.9 and $6.8 \%$, respectively. For errors of $7 \%$ in the simulated data, the recovered rate constants are $3.7 \%$ (neural network), 325\% (Simplex) and 20.5\% (Levenberg-Marquardt).

Neural network is a superior method to retrieve rate constants from simulated data with random noise, as evident from the above considerations. If simulated concentrations without random noises are employed, the three techniques have similar efficiency. Using experimental data with Levenberg-Marquardt and Simplex, the recovered constants are in total disagreement in respect to the listed in Table 1 ; despite the residual error is about $10^{-6}$ in both cases. For ill-posed problem small residual error does not mean a precise inverted result, also evident from the above consideration.

The advantage of neural networks while dealing with experimental data can be attributed to its dynamical character, since matrix inversion is not necessary in the algorithm. The neurons are adjusted to reach an objective function. The Levenberg-Marquardt algorithm requires an inversion matrix procedure, like other least squares approaches. The Simplex method also do not invert matrix, but is very sensitive with experimental error, as exemplified in the above results.

The evolution of the neuron states during the learning time shows the rate constant, $\mathrm{k}_{2}$, achieves the optimal result faster than $\mathrm{k}_{1}$ and $\mathrm{k}_{3}$. Also, it is observed that using initial guesses with error of $10 \%$ in $\mathrm{k}_{1}$ and $\mathrm{k}_{3}$, all the algorithms do not converge. This can be explained by the sensitivity analysis, which measures the capability of inversion of some parameter from experimental/simulated data. The sensitivity of Citronellol data, $C_{c}$, with respect to the rate constants, ${ }^{23}$ i.e. $S_{1}=\partial C_{c} / \partial k_{1}, S_{2}=\partial C_{c} / \partial k_{2}, S_{3}=\partial C_{c} / \partial k_{3}$ denotes the level of information 
intrinsic in the data with respect to each parameter. Figure 3 shows the sensitivity curves along the learning time. Low sensitivity values show a limited potentiality to the inversion procedure, and this explains why the convergence to obtain $\mathrm{k}_{2}$ is faster than for $\mathrm{k}_{1}$ and $\mathrm{k}_{3}$.

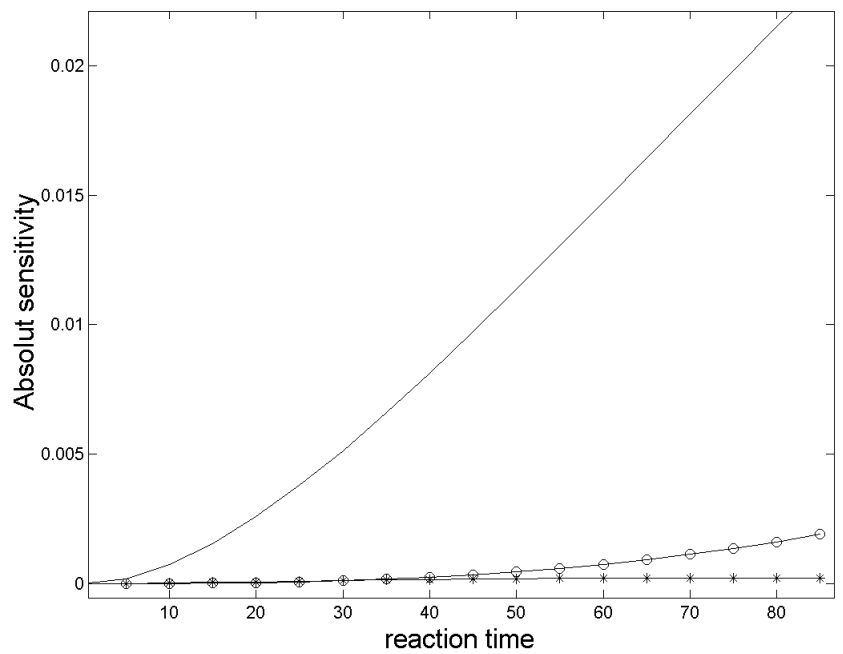

Figure 3. Reaction time evolution of Citronellol data sensitivities: $S_{1}(*), S_{2}$ (o) and $S_{3}$ (full line)

\section{CONCLUSION}

A method based on artificial neural networks has been applied to solve an inverse kinetic problem related to Citral molecule hydrogenation. Rate constants from the experimental and simulated product concentration were calculated. The accuracy of the inverse problem solution is guaranteed by the decreasing error property of the present approach and the inverted rate constants reproduce concentrations in agreement with experimental values. Analytical solutions for differential kinetics equations were also used in the present work to reproduce and discuss the results obtained by the Levenberg-Marquardt and Simplex algorithms.

The present method can be used for any set of differential equations which describe a kinetic reaction mechanism. The algorithm was numerically stable in relation to different initial conditions and also it was more robust with respect to random noises in the experimental concentration than Levenberg-Maquardt and Simplex algorithms, common inversion methods used to solve this kind of problem. The strong numerical stability of the method allows treatment of data with large experimental noises and guarantees an efficient convergence for initial conditions close to the real estimated parameters.

\section{ACKNOWLEDGEMENTS}

This work was supported by CNPq.

\section{REFERENCES}

1. Hadamard, J.; Le probleme de Cauchy et les equations aux derivees partielles lineaires hyperboliques, Hermann: Paris, 1932.

2. Furusjo, E.; Svenssonb, O.; Danielss, L.G.; Chemom. Intell. Lab. Syst. 2003, 66, 1 .

3. Zhu, Z. L.; Li, W.; Xia, J.; Anal. Chim. Acta 2004, 527, 203.

4. Alper, J. S.; Gelb, R. I.; J. Phys. Chem. 1991, 95, 104.

5. Tadi, M.; Yetter, R. A.; Int. J. Chem. Kinet. 1998, 30, 151.

6. Greco, A. M.; Direct and Inverse Methods in Nonlinear Evolution Equations, Springer: New York, 2003.

7. Lemes, N. H. T.; Borges, E.; Braga, J. P.; J. Braz. Chem. Soc. 2007, 18, 1342.

8. Lemes, N. H. T.; Borges, E.; Braga, J. P.; Chemom. Intell. Lab. Syst. 2009, 96, 84.

9. Sebastião, R. C. O.; Braga, J. P.; J. Magn. Reson. 2005, 177, 146.

10. Viterbo, V. C.; Sebastião, R. C. O.; Monteiro, R. P. G.; Magalhães, W. F.; Braga, J. P.; J. Braz. Chem. Soc. 2005, 16, 93.

11. Sebastião, R. C. O.; Lemes, N. H. T.; Virtuoso, L. S.; Braga, J. P.; Chem. Phys. Lett. 2003, 378, 406.

12. Borges, E.; Lemes, N. H. T.; Braga, J. P.; Chem. Phys. Lett. 2006, 423, 357.

13. Mäki-Arvela, P.; Denecheau, A.; Alho, K.; Wärnå, J.; Eränen, K.; Salmi, T.; Murzin, D. T.; Chem. Eng. J. 2007, 134, 268.

14. Salmi, T.; Mäki-Arvela, P.; Wärnå, J.; Eränen, K.; Alho, K.; Denecheau, A.; Murzin, D. T.; Ind. Eng. Chem. Res. 2007, 46, 3912.

15. Levenberg, K.; Quart. Appl. Math. 1944, 2, 164.

16. Marquardt, D.; SIAM J. Appl. Math. 1963, 11, 431.

17. Sebastião, R. C. O.; Braga, J. P.; Yoshida, M. I.; Thermochim. Acta 2004, 412, 107.

18. Braga, J. P.; J. Math. Chem. 2001, 29, 151.

19. Hopfield, J. J.; Proc. Natl. Acad. Sci. U.S.A. 1984, 81, 3088.

20. Hopfield, J. J.; Tank, D. W.; Biol. Cybern. 1985, 52, 141.

21. Vemuri, V.; Jang, G. S.; J. Franklin Inst. 1992, 329, 241.

22. Forsythe, G. E.; Malcolm, M. A.; Moler, C. B.; Computer Methods for Mathematical Computations, Englewood Clifs: New Jersey, 1977.

23. Lemes, N. H. T.; Sebastião, R. C. O.; Braga, J. P.; Inverse Probl. Sci. Eng. 2006, 14, 581 